\title{
Noncollinear Magnetism in Density Functional Calculations
}

\author{
Juan E. Peralta and Gustavo E. Scuseria \\ Department of Chemistry, Rice University, Houston, Texas 77005 \\ Michael J. Frisch \\ Gaussian Inc., 340 Quinnipiac St., Bldg. 40, Wallingford, Connecticut 06492
}

(Dated: February 6, 2008)

\begin{abstract}
We generalize the treatment of the electronic spin degrees of freedom in density functional calculations to the case where the spin vector variables employed in the definition of the energy functional can vary in any direction in space. The expression for the generalized exchange-correlation potential matrix elements is derived for general functionals which among their ingredients include the electron density, its gradient and Laplacian, the kinetic energy density, and non-local Hartree-Fock type exchange. We present calculations on planar Cr clusters that exhibit ground states with noncollinear spin densities due to geometrically frustrated antiferromagnetic interactions.
\end{abstract}

\section{INTRODUCTION}

Since the spin polarized formulation $\underline{1}$ of density functional theory (DFT) $)^{2,3}$ was introduced, a large number of applications have been carried out in magnetic systems. In most cases, the spin density is assumed to adopt a single direction (collinear) at each point in space that is usually taken as $z$. However, there are a number of systems where the spin density (or magnetization density) can take a more complicated structure, and vary its $d i$ rection at each point in space. These type of noncollinear structures were observed in the form of helical spin density waves or spin spirals for the ground state of $\gamma-\mathrm{Fe}, \underline{4.5}$ in geometrically frustrated systems like for instance the Kagomé antiferromagnetic lattice ${ }^{\underline{6}}$, and in systems with competing magnetic interactions such as the composite magnet $\mathrm{LaMn}_{2} \mathrm{Ge}_{2}{ }^{7}$ and $\mathrm{Fe}_{0.5} \mathrm{Co}_{0.5} \mathrm{Si}, \frac{8}{.}$

Several papers dealing with noncollinear spin density in DFT calculations have been published in the literature. The pioneer work of Kübler and coworkers ${ }^{9}$ for the noncollinear local spin density approximation (LSDA) was later followed by a number of independent implementations and applications. Most of these implementations were carried out using periodic boundary conditions and plane waves, and are based either on the $\operatorname{LSDA}^{10,11}$ or on a generalized gradient approximation (GGA) $\stackrel{12}{*}$ Yamanaka and coworkers developed a generalized DFT code based on Gaussian type orbitals. $\stackrel{13}{=}$ Some noncollinear DFT calculations have been published dealing with magnetic crystals,,$\frac{5,10}{14}$ and with fourth period transition metal clusters $, 11,14,15,16$ In all cases, the realization of the LSDA and GGA employed in noncollinear calculations is the same as that developed for collinear spin systems.

Different parametrizations of the exchange-correlation energy $\left(E_{x c}\right)$ have been proposed beyond the LSDA and the GGA, incorporating more ingredients in the definition of $E_{x c}$. The third rung in this hierarchy $\frac{17}{1}$ is the meta-GGA, which includes the kinetic energy density as a functional ingredient. Also, hybrid density functionals, which contain a portion of Hartree-Fock type exchange, can be regarded as belonging to the fourth rung (hyperGGA) in this picture. $\stackrel{17}{ }$ The purpose of this paper is to provide a consistent generalization for the treatment of noncollinear spin variables in DFT calculations beyond the LSDA.

\section{THEORY}

To allow for noncollinear spin states in density functional calculations, we start by introducing twocomponent spinors as Kohn-Sham (KS) orbitals:

$$
\Psi_{i}=\left(\begin{array}{c}
\psi_{i}^{\alpha} \\
\psi_{i}^{\beta}
\end{array}\right)
$$

where $\psi_{i}^{\alpha}$ and $\psi_{i}^{\beta}$ are spatial orbitals that can be expanded in a linear combination of atomic orbitals,

$$
\psi_{i}^{\sigma}(\mathbf{r})=\sum_{\mu} c_{\mu i}^{\sigma} \phi_{\mu}(\mathbf{r})(\sigma=\alpha, \beta) .
$$

Using the KS formulation, the electronic energy is partitioned into four contributions:

$$
E=E_{T}+E_{N}+E_{J}+E_{x c},
$$

where $E_{T}$ is the kinetic energy, $E_{N}$ is the nuclear-electron interaction energy, $E_{J}$ is the classical electron-electron Coulomb repulsion energy, and $E_{x c}$ is the exchangecorrelation (XC) energy. Searching for stationary solutions of $E$ is equivalent to solving the KS equations, which in terms of two-component spinors $\Psi_{i}$ read:

$$
\left(T+V_{N}+J+V_{x c}\right) \Psi_{i}=\epsilon_{i} \Psi_{i},
$$

where $T=-1 / 2 \nabla^{2}$ is the kinetic energy operator, $V_{N}$ is the external electron-nuclear potential, $J$ is the Coulomb operator, and $V_{x c}$ is the exchange-correlation (XC) potential. We shall here refer to the KS equations in a two-component spinor basis as the generalized KS (GKS) equations. Since $T, V_{N}$, and $J$ are diagonal in the twodimensional spin space, the only term in Eq. 4 that couples $\psi_{i}^{\alpha}$ and $\psi_{i}^{\beta}$ is $V_{x c}$ (the spin-orbit operator, present 
in a relativistic Hamiltonian, also couples the two spinor components). The potential $V_{x c}$ depends on the choice of $E_{x c}$ and therefore the coupling between $\psi_{i}^{\alpha}$ and $\psi_{i}^{\beta}$ in the non-relativistic GKS equations depends exclusively on $E_{x c}$.

Let us first recall the standard formulation of $E_{x c}$ commonly employed in (collinear) unrestricted KS (UKS) calculations. The general expression of $E_{x c}$ for a hybrid case can be cast as: $\stackrel{18}{1}$

$$
E_{x c}=a E_{x}^{D F A}+E_{c}^{D F A}+(1-a) E_{x}^{H F},
$$

where $E_{x}^{D F A}$ and $E_{c}^{D F A}$ are the exchange-correlation contributions to the energy at some (semi)local density functional approximation (DFA), respectively, $E_{x}^{H F}$ is the Hartree-Fock type exchange energy, and $a$ is a mixing parameter $(0 \leq a \leq 1)$. The functional forms of $E_{x}^{D F A}$ and $E_{c}^{D F A}$ (as well as the parameter $a$ ) depend, of course, on the choice of the functional employed in the actual calculation. A general expression for $E_{x c}^{D F A}=a E_{x}^{D F A}+E_{c}^{D F A}$ can be written as

$$
E_{x c}^{D F A}=\int d^{3} r f(\mathcal{Q}),
$$

where $\mathcal{Q}$ is a set of variables (included in the definition of $\left.E_{x c}\right)$ :

$$
\mathcal{Q} \equiv\left\{n_{\alpha}, n_{\beta}, \nabla n_{\alpha}, \nabla n_{\beta}, \tau_{\alpha}, \tau_{\beta}, \nabla^{2} n_{\alpha} \nabla^{2} n_{\beta}\right\} .
$$

Here $n_{\alpha}$ and $n_{\beta}$ are the $\alpha$ and $\beta$ electron densities, and $\tau_{\alpha}$ and $\tau_{\beta}$ are the $\alpha$ and $\beta$ kinetic energy densities, respectively, representing the "up" and "down" components along the $z$ axis.

On the other hand, in the noncollinear case, where the vector component of the local variables employed in the definition of $E_{x c}$ can point in any direction, $E_{x c}^{D F A}$ can be generalized as follows,

$$
E_{x c}^{N C}=\int d^{3} r f(\widetilde{\mathcal{Q}}),
$$

where

$$
\widetilde{\mathcal{Q}} \equiv\left\{n_{+}, n_{-}, \nabla n_{+}, \nabla n_{-}, \tau_{+}, \tau_{-}, \nabla^{2} n_{+} \nabla^{2} n_{-}\right\} .
$$

Here the subindices + and - refer to variables expressed in a local reference frame along the local spin quantization axis. The definition of these variables is given in detail in Section II. Note that by replacing $\mathcal{Q}$ by $\widetilde{\mathcal{Q}}$, we have only added degrees of freedom to the local variables in such a way that they are compatible with any arbitrary choice of the local spin axis. The dependence of $f$ (and therefore $E_{x c}^{N C}$ ) on these variables remains unchanged.

Two conditions must be satisfied by the set of variables in $\widetilde{\mathcal{Q}}$. First, we should recover the standard collinear case if we allow spin polarization only in one direction, and therefore replace the labels + and - in $E_{x c}^{N C}$ by $\alpha$ and $\beta$, respectively. In other words, noncollinear GKS solutions should coincide with collinear solutions obtained with the standard UKS approximation in cases where the ground state solution is collinear. Second, for spinindependent Hamiltonians, any arbitrary choice (other than $z$ ) of the spin quantization axis should leave the energy unchanged. This means that any rigid rotation of all local reference frames should not change the total energy.

We therefore assume that $E_{x c}^{N C}$ depends on the local variables + and - in the same manner as in the standard collinear case, and that $E_{x c}^{N C}$ must be invariant under rigid rotations of the spin quantization axis. This is, of course, not the most general possibility to define energy functionals for noncollinear magnetic systems $\underline{\underline{19}}$

For practical applications, it is necessary to evaluate the $\mathrm{XC}$ potential matrix to be employed in the solution of the GKS equations. These matrix elements in a set of localized orbitals $\left\{\phi_{\xi}\right\}$ can be written as:

$$
\begin{aligned}
\left(V_{x c}^{N C}\right)_{\mu \nu} & =\int d^{3} r \frac{\partial f(\widetilde{\mathcal{Q}})}{\partial P_{\mu \nu}} \\
& =\sum_{p, q \in \widetilde{\mathcal{Q}}} \int d^{3} r \frac{\partial f(\widetilde{\mathcal{Q}})}{\partial q} \frac{\partial q}{\partial p} \frac{\partial p}{\partial P_{\mu \nu}},
\end{aligned}
$$

where $P_{\mu \nu}$ are matrix elements of the generalized density matrix,

$$
P_{\mu \nu}=\sum_{i \in o c c}\left(\begin{array}{ccc}
c_{\mu i}^{\alpha} c_{\nu i}^{\alpha *} & c_{\mu i}^{\alpha} c_{\nu i}^{\beta *} \\
c_{\mu i}^{\beta} c_{\nu i}^{\alpha *} & c_{\mu i}^{\beta} c_{\nu i}^{\beta *}
\end{array}\right),
$$

and $p$ represents variables that are linear in $P_{\mu \nu}$. The derivative $\partial f / \partial q$ remains the same as in the collinear case. The rest of this section is devoted to the definition of the variables $q \in \widetilde{\mathcal{Q}}$ in the local reference frame, and to the evaluation of $\left(V_{x c}^{N C}\right)_{\mu \nu}$.

\section{A. Density}

The main ingredient for the construction of $E_{x c}^{N C}$ in the LSDA is the generalized density, $\bar{n}$, which can be written in a two-component spin space as

$$
\bar{n}=\frac{1}{2}(n+\mathbf{m} \cdot \boldsymbol{\sigma})=\frac{1}{2}\left(\begin{array}{cc}
n+m_{z} & m_{x}-i m_{y} \\
m_{x}+i m_{y} & n-m_{z}
\end{array}\right),
$$

where $n$ is the electron density, $\mathbf{m}=\left(m_{x}, m_{y}, m_{z}\right)$ is the spin density (magnetization) vector, and $\boldsymbol{\sigma}=$ $\left(\sigma_{x}, \sigma_{y}, \sigma_{z}\right)$ are the Pauli matrices. In terms of twocomponent spinors $\Psi_{i}, n$ and $\mathbf{m}$ are defined as

$$
n(\mathbf{r})=\sum_{i \in o c c} \Psi_{i}^{\dagger}(\mathbf{r}) \Psi_{i}(\mathbf{r}),
$$

and

$$
m_{k}(\mathbf{r})=\sum_{i \in o c c} \Psi_{i}^{\dagger}(\mathbf{r}) \sigma_{k} \Psi_{i}(\mathbf{r}) \quad(k=x, y, z) .
$$


Using Eqs. 1, 2, and 11, it is straightforward to express $n$ and $\mathbf{m}$ as a linear combination of matrix elements of the generalized density matrix $P_{\mu \nu}$.

A local reference system where the generalized density $\bar{n}$ is diagonal can be obtained by rotating $\bar{n}$ into $\bar{n}^{\prime}$,

$$
\bar{n}^{\prime}=\left(\begin{array}{cc}
n_{+} & 0 \\
0 & n_{-}
\end{array}\right)
$$

where

$$
n_{ \pm}=\frac{1}{2}(n \pm m)=\frac{1}{2}\left(n \pm \sqrt{m_{x}^{2}+m_{y}^{2}+m_{z}^{2}}\right)
$$

are the eigenvalues of $\bar{n}$. The densities $n_{+}$and $n_{-}$can be regarded as the local analogous of $n_{\alpha}$ and $n_{\beta}$. The potential $V_{x c}^{N C}$ is

$$
\begin{aligned}
V_{x c}^{N C} & =\frac{\delta E_{x c}^{N C}}{\delta \bar{n}} \\
& =\frac{1}{2}\left(f^{+}+f^{-} \hat{\mathbf{m}} \cdot \boldsymbol{\sigma}\right),
\end{aligned}
$$

where

$$
f^{ \pm}=\frac{\partial f}{\partial n_{+}} \pm \frac{\partial f}{\partial n_{-}}
$$

and $\hat{\mathbf{m}}=\mathbf{m} / m$ is the unit vector in the direction of $\mathbf{m}$. The matrix elements of $V_{x c}^{N C}$ can be evaluated straightforwardly as

$$
\left(V_{x c}^{N C}\right)_{\mu \nu}=\int d^{3} r \phi_{\mu} V_{x c}^{N C} \phi_{\nu} .
$$

The potential $V_{x c}^{N C}$ in Eq. 17 can be split into two contributions,

$$
V_{x c}^{N C}=\mathcal{E}_{x c}^{N C}+\boldsymbol{\sigma} \cdot \mathcal{B}_{x c}^{N C},
$$

where $\mathcal{E}_{x c}^{N C}=f^{+} / 2$ can be interpreted as a scalar (electrostatic) potential and $\mathcal{B}_{x c}^{N C}=f^{-} \hat{\mathbf{m}} / 2$ as a spin-dependent (magnetic) potential, which for the LSDA is always parallel to $\mathbf{m}$. It is worth mentioning that in the limit of no magnetization $(m \rightarrow 0), f^{-} \rightarrow 0$ and therefore $\mathcal{B}_{x c}^{N C} \rightarrow \mathbf{0}$, recovering the non-magnetic case.

\section{B. Density gradients}

Let us consider next GGA energy functionals. In this case the new ingredients in $E_{x c}^{N C}$ are $\nabla n_{+}$and $\boldsymbol{\nabla} n_{-}$, whose Cartesian component $j(j=x, y, z)$ is

$$
\begin{aligned}
\nabla_{j} n_{ \pm} & =\frac{\partial n_{ \pm}}{\partial j} \\
& =\frac{1}{2}\left(\nabla_{j} n \pm \frac{1}{m} \sum_{k=x, y, z} m_{k} \nabla_{j} m_{k}\right) .
\end{aligned}
$$

We note in passing that this family of density functionals usually depends on the gradient of the density through the auxiliary quantities ${ }^{20}$

$$
\gamma_{a b}=\nabla n_{a} \cdot \nabla n_{b} \quad a, b=+,-, .
$$

The matrix elements of $V_{x c}^{N C}$ are

$$
\begin{aligned}
\left(V_{x c}^{N C}\right)_{\mu \nu}= & \sum_{j=x, y, z} \int \frac{\partial f}{\partial\left(\nabla_{j} n\right)} \nabla_{j}\left(\phi_{\mu} \phi_{\nu}\right) d^{3} r \\
& +\sum_{j, k=x, y, z} \int \frac{\partial f}{\partial\left(\nabla_{j} m_{k}\right)} \sigma_{k} \nabla_{j}\left(\phi_{\mu} \phi_{\nu}\right) d^{3} r \\
& +\sum_{k=x, y, z} \int \frac{\partial f}{\partial m_{k}} \sigma_{k}\left(\phi_{\mu} \phi_{\nu}\right) d^{3} r
\end{aligned}
$$

where the first term on the right-hand side of Eq. 23 contributes to the scalar potential $\mathcal{E}_{x c}^{N C}$, and the second and last terms add to the magnetic potential $\mathcal{B}_{x c}^{N C}$. The last term on the right-hand side of Eq. 23] arises from the fact that $\boldsymbol{\nabla} n_{ \pm}$depends on $\mathbf{m}$ (Eq. 21). Applying the chain rule, and making use of Eq. 21, the derivatives of $f$ in Eq. 23 can be expressed as (we do not consider here derivatives of $f$ arising from $\partial f / \partial n_{ \pm}$since they where considered in the previous section)

$$
\begin{gathered}
\frac{\partial f}{\partial\left(\nabla_{j} n\right)}=\frac{1}{2} g_{j}^{+}, \\
\frac{\partial f}{\partial\left(\nabla_{j} m_{k}\right)}=\frac{1}{2} \frac{m_{k}}{m} g_{j}^{-},
\end{gathered}
$$

and

$$
\frac{\partial f}{\partial m_{k}}=\sum_{l=x, y, z} \frac{1}{2}\left\{\frac{\nabla_{l} m_{k}}{m} g_{l}^{+}-\frac{m_{k}}{m^{2}} g_{l}^{-} \nabla_{l} m\right\},
$$

where we have defined

$$
g_{k}^{ \pm}=\frac{\partial f}{\partial\left(\nabla_{k} n_{+}\right)} \pm \frac{\partial f}{\partial\left(\nabla_{k} n_{-}\right)} .
$$

Alternatively, one can calculate $V_{x c}^{N C}$ as the functional derivative

$$
\begin{aligned}
V_{x c}^{N C} & =\frac{\delta E_{x c}^{N C}}{\delta \bar{n}} \\
& =\frac{\delta E_{x c}^{N C}}{\delta n}+\sum_{i} \frac{\delta E_{x c}^{N C}}{\delta m_{i}} \sigma_{i} .
\end{aligned}
$$

Using the chain rule for functional derivatives, $\delta E_{x c}^{N C} / \delta m_{i}$ can be written as

$$
\frac{\delta E_{x c}^{N C}}{\delta m_{i}}=\frac{1}{2}\left(\frac{\delta E_{x c}^{N C}}{\delta n_{+}}-\frac{\delta E_{x c}^{N C}}{\delta n_{-}}\right) \frac{m_{i}}{m} .
$$

Multiplying Eq. 29 by $\phi_{\mu} \phi_{\nu}$, integrating over all space and applying integration by parts, we obtain Eq. 23. Therefore, from our definition of $E_{x c}^{N C}$, the contribution to the XC magnetic field $\mathcal{B}_{x c}^{N C}$ from Eq. 28 is always parallel to $\mathbf{m}$. However, this is not necessarily the case for a general form of a GGA functional, as shown by Capelle and coworkers $\underline{\underline{21}}$

One issue that is worth addressing is how our formulation differs from previous noncollinear generalizations 
of the GGA. In our case, we employ $\nabla n_{ \pm}$as defined in Eq. 21, and therefore the ingredients used in $E_{x c}^{N C}$ are strictly the gradients of the quantities $n_{ \pm}$. Other implementations ${ }^{12,22}$ employ either $\nabla m$ or the $z$ component of the projection of $\boldsymbol{\nabla} m_{k}$ onto $\mathbf{m}$, therefore imposing the constraint of $\mathcal{B}_{x c}^{N C}$ being parallel to $\mathbf{m}$. Here as in the LSDA case, in the limit of no magnetization $(m \rightarrow 0$ and $\boldsymbol{\nabla} m \rightarrow \mathbf{0}), g_{k}^{-} \rightarrow 0$ for $k=x, y, z$ and hence $\mathcal{B}_{x c}^{N C} \rightarrow \mathbf{0}$, recovering the non-magnetic case.

\section{Kinetic energy density}

To deal with kinetic energy density contributions, we can proceed in analogy to Sec. II A and define a generalized kinetic energy density:

$$
\bar{\tau}=\frac{1}{2}(\tau+\mathbf{u} \cdot \boldsymbol{\sigma})=\frac{1}{2}\left(\begin{array}{cc}
\tau+u_{z} & u_{x}-i u_{y} \\
u_{x}+i u_{y} & \tau-u_{z}
\end{array}\right),
$$

where $\tau$ and $\mathbf{u}$ can be written in terms of two-component spinors as

$$
\tau(\mathbf{r})=\frac{1}{2} \sum_{i \in o c c}\left(\nabla \Psi_{i}(\mathbf{r})\right)^{\dagger} \cdot \nabla \Psi_{i}(\mathbf{r})
$$

and

$$
u_{k}(\mathbf{r})=\frac{1}{2} \sum_{i \in o c c}\left(\nabla \Psi_{i}(\mathbf{r})\right)^{\dagger} \sigma_{k} \cdot \nabla \Psi_{i}(\mathbf{r}) \quad(k=x, y, z)
$$

Comparing $\bar{\tau}$ (Eq. 30) and $\bar{n}$ (Eq. 12) one is tempted to define $\tau_{+}$and $\tau_{-}$as the local eigenvalues of $\bar{\tau}$. However, this choice would lead to a different local reference frame than the one used for $\bar{n}$, and therefore collinear solutions obtained in this way will not necessarily be the same as those obtained with standard unrestricted KS calculations. To avoid this problem, we have chosen the following definition for $\tau_{+}$and $\tau_{-}$,

$$
\tau_{ \pm}=\frac{1}{2}(\tau \pm \hat{\mathbf{m}} \cdot \mathbf{u})
$$

which is equivalent to locally projecting $\mathbf{u}$ onto the axis defined by $\mathbf{m}$. Using this choice for $\tau_{+}$and $\tau_{-}$, the contribution to the $\mathrm{XC}$ potential matrix elements can be written as

$$
\begin{aligned}
\left(V_{x c}^{N C}\right)_{\mu \nu}= & \sum_{j=x, y, z} \int \frac{\partial f}{\partial \tau}\left(\nabla_{j} \phi_{\mu} \nabla_{j} \phi_{\nu}\right) d^{3} r \\
& +\sum_{j, k=x, y, z} \int \frac{\partial f}{\partial u_{k}} \sigma_{k}\left(\nabla_{j} \phi_{\mu} \nabla_{j} \phi_{\nu}\right) d^{3} r \\
& +\sum_{k=x, y, z} \int \frac{\partial f}{\partial m_{k}} \sigma_{k}\left(\phi_{\mu} \phi_{\nu}\right) d^{3} r .
\end{aligned}
$$

The partial derivatives of $f$ in Eq. 34 can be expressed in terms of the derivatives of $f$ with respect to $\tau_{+}$and $\tau$ - as

$$
\frac{\partial f}{\partial \tau}=\frac{1}{2} h^{+}
$$

$$
\frac{\partial f}{\partial u_{k}}=\frac{m_{k}}{2 m} h^{-}
$$

and

$$
\frac{\partial f}{\partial m_{k}}=\frac{1}{2 m} h^{-} \sum_{j=x, y, z} u_{j}\left(\delta_{j k}-\frac{m_{j} m_{k}}{m^{2}}\right),
$$

where

$$
h^{ \pm}=\frac{\partial f}{\partial \tau_{+}} \pm \frac{\partial f}{\partial \tau_{-}} .
$$

The first term on the right-hand side of Eq. 34 contributes to the scalar potential $\mathcal{E}_{x c}^{N C}$, while the second and third terms are spin dependent and therefore contribute to $\mathcal{B}_{x c}^{N C}$. In the limit where $m \rightarrow 0$ then $|\mathbf{m} \cdot \mathbf{u}| \rightarrow 0$, and therefore $\mathcal{B}_{x c}^{N C} \rightarrow \mathbf{0}$ since $h^{-} \rightarrow 0$.

\section{Laplacian of the density}

The dependence of $E_{x c}^{D F A}$ with the Laplacian of the density can be generalized for the noncollinear case through $\nabla^{2} n_{ \pm}$, which in terms of $n, \mathbf{m}$, and their derivatives can be written as:

$$
\begin{aligned}
\nabla^{2} n_{ \pm}= & \frac{1}{2}\left\{\nabla^{2} n \pm \sum_{k=x, y, z}\left(\frac{m_{k}}{m} \nabla^{2} m_{k}+\sum_{j=x, y, z}\left(\frac{\left(\nabla_{j} m_{k}\right)^{2}}{m}\right.\right.\right. \\
& \left.\left.\left.-\sum_{l=x, y, z} \frac{m_{l} m_{k} \nabla_{j} m_{l} \nabla_{j} m_{k}}{m^{3}}\right)\right)\right\}
\end{aligned}
$$

Three types of contributions to the XC potential arise in this case, since $\nabla^{2} n_{+}$and $\nabla^{2} n_{-}$depend on $\nabla^{2} n$, $\nabla^{2} m_{k}, m_{k}$, and $\nabla m_{k}$ (Eq. 39).

$$
\begin{aligned}
\left(V_{x c}^{N C}\right)_{\mu \nu}= & \int\left(t^{+}+t^{-} \boldsymbol{\sigma} \cdot \hat{\mathbf{m}}\right) \nabla^{2}\left(\phi_{\mu} \phi_{\nu}\right) d^{3} r \\
& +\sum_{j=x, y, z} \int\left(t^{+}+t^{-} \boldsymbol{\sigma} \cdot \hat{\mathbf{m}}\right)\left(\nabla_{j} \phi_{\mu} \nabla_{j} \phi_{\nu}\right) d^{3} r \\
& +\sum_{k, j=x, y, z} \int \frac{t^{-} \sigma_{k}}{2} \frac{\partial\left(\nabla^{2} m\right)}{\partial\left(\nabla_{j} m_{k}\right)}\left(\nabla_{j} \phi_{\mu} \nabla_{j} \phi_{\nu}\right) d^{3} r \\
& +\sum_{k=x, y, z} \int \frac{t^{-} \sigma_{k}}{2} \frac{\partial\left(\nabla^{2} m\right)}{\partial m_{k}}\left(\phi_{\mu} \phi_{\nu}\right) d^{3} r
\end{aligned}
$$

where

$$
t^{ \pm}=\frac{\partial f}{\partial\left(\nabla^{2} n_{+}\right)} \pm \frac{\partial f}{\partial\left(\nabla^{2} n_{-}\right)}
$$

The derivatives of $\nabla^{2} m$ with respect to the linear variables can be obtained from Eq. 39. In the limit of no magnetization where $m \rightarrow 0$ and $\nabla^{2} m \rightarrow 0$, all contributions to $\mathcal{B}_{x c}^{N C}$ are zero since $t^{-} \rightarrow 0$. 


\section{E. Hartree-Fock type exchange}

The HF type exchange contribution to $\left(V_{x c}\right)_{\mu \nu}$, needed for hybrid density functional calculations, can be generalized in terms of two-component spinors as

$$
K_{\mu \nu}^{\sigma \sigma^{\prime}}=\sum_{\lambda \xi} P_{\lambda \xi}^{\sigma \sigma^{\prime}}(\mu \lambda \mid \xi \nu),
$$

where $P_{\lambda \xi}^{\sigma \sigma^{\prime}}$ are the spin blocks of the generalized density matrix in Eq. (11). The notation $(\mu \nu \mid \xi \lambda)$ has been introduced for the two electron integrals in the AO basis set. This is analogous to the generalized unrestricted HF (GUHF) approximation. ${ }^{23}$ The evaluation of the four matrix blocks of $K_{\mu \nu}^{\sigma \sigma^{\prime}}$ is carried out by splitting $P_{\lambda \xi}^{\sigma \sigma^{\prime}}$ into real and imaginary parts, and symmetric and antisymmetric components. The symmetric imaginary and antisymmetric real contributions to $K^{\alpha \alpha}$ and $K^{\beta \beta}$ are zero because of the hermiticity requirement of the KohnSham (or Hartree-Fock) Hamiltonian. Therefore, a total of eight HF exchange blocks need to be computed, four of them are symmetric and four are antisymmetric.

\section{IMPLEMENTATION}

We have implemented the SCF solution of the GKS equations in the Gaussian suite of programs. ${ }^{24}$ Molecular spinors (Eq. 2) are spanned in terms of atomic Gaussian orbitals using a set of complex coefficients $c_{\mu i}^{\sigma}$. These coefficients are employed to construct the generalized density matrix $P_{\mu \nu}$ (Eq. 11), from which the Hartree-Fock type exchange matrix can be calculated, as well as all the variables needed for the numerical quadrature employed in the evaluation of $\left(V_{x c}^{N C}\right)_{\mu \nu}$. To accelerate the SCF convergence, we have generalized the direct inversion of the iterative subspace (DIIS) ${ }^{25}$ and the energy-based DIIS ${ }^{26}$ techniques for two-component complex spinors.

In a GKS calculation, the spin density of the system is fully unconstrained, and is thus allowed to change in any arbitrary spatial direction. For instance, in a simple calculation of the (nonrelativistic) ground state of the hydrogen atom using LSDA, one can obtain an infinite manifold of solutions with the same total energy but different orientation of the spin density. These solutions are just linear combinations of the two degenerate linearly independent solutions.

We have verified for a representative sample of functionals that for cases with ground state collinear solutions, the GKS solution for different choices of the quantization axis gives the same total energy as in a collinear UKS calculation. We have also verified that the calculated electric dipole moment evaluated as finite differences agrees with the expectation value of the electric dipole operator, satisfying the Hellmann-Feynman theorem.

The third term on the right-hand side of Eq. 23 leads to instabilities in the numerical integration due to the

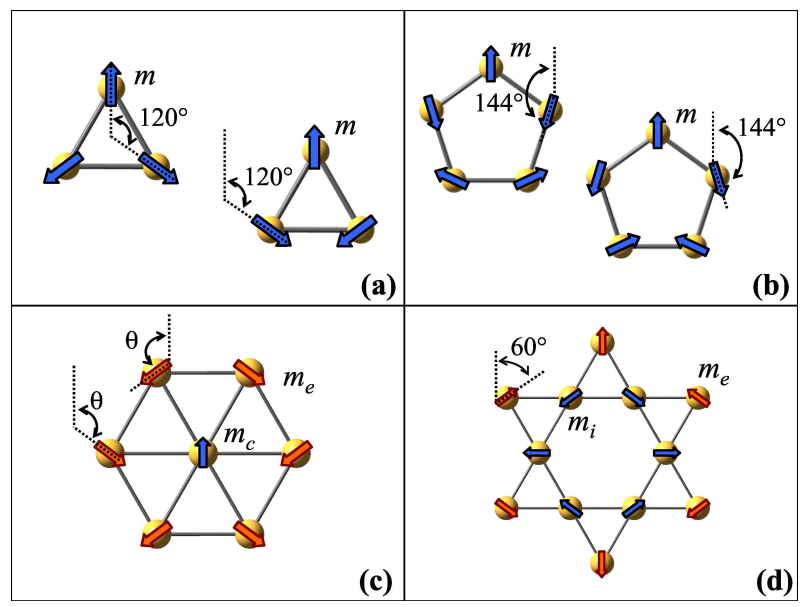

FIG. 1: Schematic representation of the $\mathrm{Cr}_{3}, \mathrm{Cr}_{5}, \mathrm{Cr}_{7}$, and $\mathrm{Cr}_{12}$ clusters employed in our tests. The arrows represent the magnetization orientation on each atom as qualitatively obtained in our calculations. For $\mathrm{Cr}_{3}$ and $\mathrm{Cr}_{5}$, two different chiralities were considered.

presence of $\boldsymbol{\nabla}\left(m_{i} / m\right)$, which is exactly zero for collinear spin densities but may present significant oscillations for spin densities that are slightly noncollinear. For some cases, this prevented converging the total SCF energy better than $10^{-6}$ hartree, which is not enough for our standard accurate convergence criteria. To avoid this problem, we discard contributions to Eq. 23 from grid points where the magnetization helicity, defined as $m_{h}=$ $\mathbf{m} \cdot(\boldsymbol{\nabla} \times \mathbf{m})$, is less than a certain threshold $\left(m_{h}=0\right.$ in collinear cases). In our tests, a cutoff value of $m_{h}<10^{-6}$ worked reasonably well.

\section{RESULTS}

In order to test our GKS code, we have chosen a set of planar Cr clusters where the ground state is expected to exhibit noncollinear spin density arising from geometrically frustrated antiferromagnetic coupling (as in a Heisenberg spin Hamiltonian model) between neighboring $\mathrm{Cr}$ atoms. In Fig. 1, we show a scheme of the $\mathrm{Cr}_{3}$ $\left(C_{3 v}\right), \mathrm{Cr}_{5}\left(C_{5 v}\right), \mathrm{Cr}_{7}\left(C_{6 v}\right)$, and $\mathrm{Cr}_{12}\left(C_{6 v}\right)$ clusters and their resulting magnetic structures obtained in this work. In all cases, we have set the $\mathrm{Cr}-\mathrm{Cr}$ bond length to 3.70 Bohr in our calculations. This allows us to compare the direct effect of each functional on the magnetization.

All calculations were carried out using a Ne core energy-consistent relativistic effective core potential (RECP) from the Stuttgart/Cologne group (Ref. 27). We have employed a polarized triple- $\zeta$ Gaussian basis set consisting of $8 s 7 p 6 d 1 f$ functions contracted to $6 s 5 p 3 d 1 f:^{27}$ Even though our implementation offers the possibility of including the spin-orbit operator, either using RECPs or in an all-electron framework, we have chosen not to include the spin-orbit interaction in the present test calculations. Atomic magnetic moments are calcu- 
TABLE I: Atomic magnetic moments (in Bohr magnetons, $\mu_{B}$ ) and $\left\langle S^{2}\right\rangle$ (in $\mu_{B}^{2}$ ) of Cr clusters calculated using different energy functionals. See Fig. 1 for a scheme of the spin density configurations.

\begin{tabular}{llrrrrr}
\hline \hline \multirow{2}{*}{ Cluster } & \multirow{2}{*}{ Property } & \multicolumn{5}{c}{ Method } \\
\cline { 3 - 7 } & & LSDA & PBE & TPSS & PBEh & GUHF \\
\hline $\mathrm{Cr}_{3}\left(C_{3 v}\right)$ & $m$ & 1.44 & 1.66 & 1.93 & 2.40 & 2.95 \\
& $\left\langle S^{2}\right\rangle$ & 3.25 & 3.87 & 4.71 & 6.32 & 8.11 \\
\hline $\mathrm{Cr}_{5}\left(C_{5 v}\right)$ & $m$ & 1.61 & 1.84 & 2.07 & 2.47 & 2.91 \\
& $\left\langle S^{2}\right\rangle$ & 5.21 & 6.21 & 7.38 & 9.78 & 12.47 \\
\hline $\mathrm{Cr}_{7}\left(C_{6 v}\right)$ & $m_{c}$ & 0.18 & 0.21 & 0.31 & 2.09 & 2.36 \\
& $m_{e}$ & 0.24 & 0.89 & 1.29 & 2.33 & 2.87 \\
& $\theta$ (deg.) & 143 & 103 & 100 & 105 & 107 \\
& $\left\langle S^{2}\right\rangle$ & 2.08 & 4.02 & 5.80 & 14.60 & 22.57 \\
\hline $\mathrm{Cr}_{12}\left(C_{6 v}\right)$ & $m_{i}$ & 0.72 & 0.84 & 1.03 & 1.86 & 2.15 \\
& $m_{e}$ & 1.24 & 1.50 & 1.72 & 2.28 & 2.80 \\
& $\left\langle S^{2}\right\rangle$ & 5.73 & 7.60 & 9.67 & 17.71 & 22.82 \\
\hline \hline
\end{tabular}

lated according to Mulliken population analysis. The expectation value $\left\langle S^{2}\right\rangle$ is evaluated in all DFT cases as for the GUHF determinant.

We have chosen one representative functional from each class of functionals discussed in Section II. For the LSDA, we employ LDA (Dirac) exchange and the parametrization of Wosko, Wilk, and Nusair ${ }^{28}$ for correlation (SVWN5); for the GGA we use the functional of Perdew, Burke, and Ernzerhof (PBE) $\stackrel{29}{\Rightarrow}$ for the metaGGA we use the functional developed by Tao, Perdew, Staroverov, and Scuseria (TPSS) $; \underline{\underline{30}}$ and as a representative hybrid functional we use $\mathrm{PBEh}^{31}$ ( $\mathrm{PBE}$ hybrid, also refer to as $\mathrm{PBE} \mathrm{PBE}^{32}$ and $\mathrm{PBE} 0^{33}$ in the literature). For comparison, we also present results for the GUHF case.

For $\mathrm{Cr}_{3}$ and $\mathrm{Cr}_{5}$, we were able to verify that the two chiral magnetic structures (Fig. 1a and Fig. 1b, respectively) have the same total energy. These two chiral magnetic states can be thought of as a product of a reflection of the spin density pseudovector in a molecular symmetry plane. For $\mathrm{Cr}_{3}$ and $\mathrm{Cr}_{5}$, we have found that starting the SCF procedure from different initial guesses always leads to coplanar spin densities, although the plane containing the spin density does not necessarily coincide with the plane containing the nuclei since the spin density can arbitrarily rotate without changing the total energy. We therefore have chosen to constrain the spin magnetization to the plane containing the nuclei for the rest of our tests.

In Figs. 2 and 3, we present a plot of the PBE spin density in the plane containing the nuclei for $\mathrm{Cr}_{3}$ and $\mathrm{Cr}_{5}$, respectively. The white (low spin polarization) holes at the nuclear positions are a consequence of the pseudopotential approximation. The red zones surrounding the nuclei correspond to high spin polarization regions. Four lobes can be distinguished around each atomic center, which is a signature of the spin polarization of the $d$ orbitals.

From Figs. 2] and 3, it can also be seen that the magne-

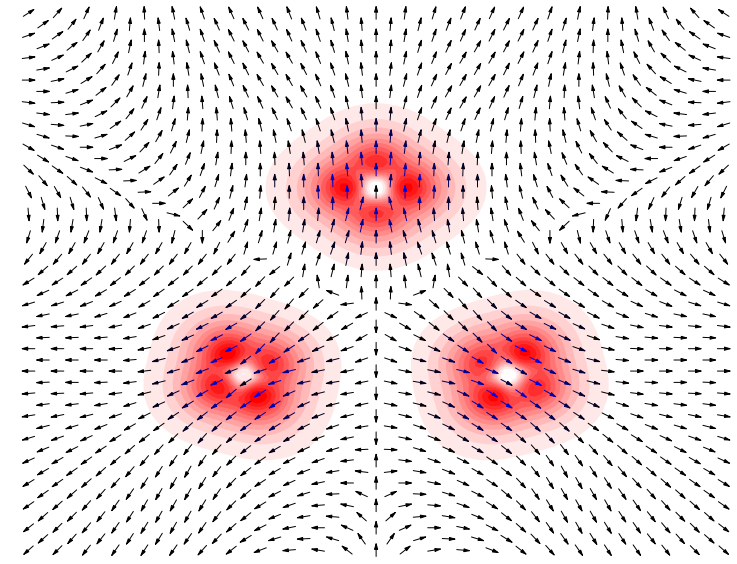

(a)

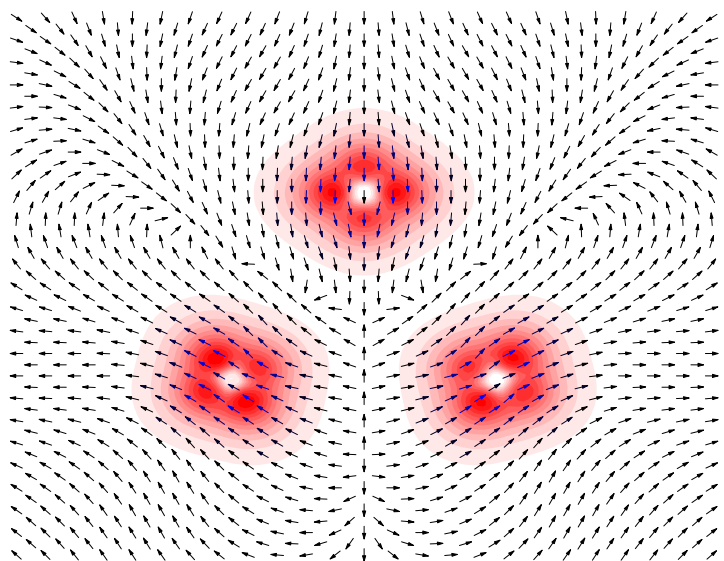

(b)

FIG. 2: Magnetization plot for $\mathrm{Cr}_{3}$ obtained in a PBE calculation. The arrows show the direction of the spin polarization $(\mathbf{m} / m)$ in the plane containing the nuclei, whereas the spin modulus $m$ is represented in red. The top (a) and bottom (b) panels show two energetically degenerate configurations with + and - chiralities, respectively.

tization tends to be collinear in the atomic regions. Inside these atomic domains, the magnetization angle changes smoothly whereas it changes abruptly at the domain boundary. This was also observed in Fe clusters 11 and in unsupported Cr monolayers in the $120^{\circ}$ Néel state. ${ }^{12}$ Spin density plots obtained using density functionals other than PBE do not differ qualitatively from these plots. However, the magnitude of the spin polarization does, as it is discussed below.

In Table I] we summarize the results obtained for Cr clusters with the different functionals. In all cases, the atomic magnetization increases systematically when going from LSDA $\rightarrow \mathrm{PBE} \rightarrow \mathrm{TPSS} \rightarrow \mathrm{PBEh} \rightarrow \mathrm{GUHF}$. The value of $\left\langle S^{2}\right\rangle$ follows the same trend, and it can be taken as a measure of the total magnetization. As a remark, 


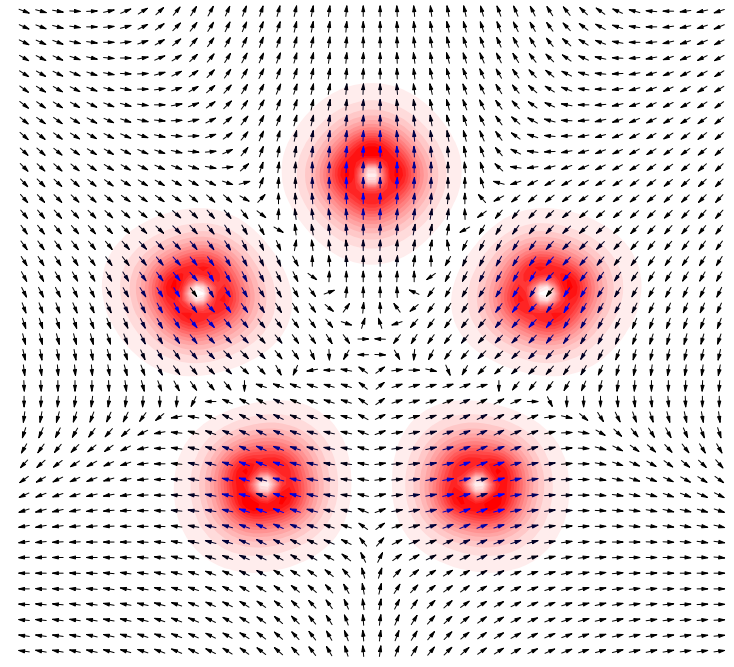

(a)

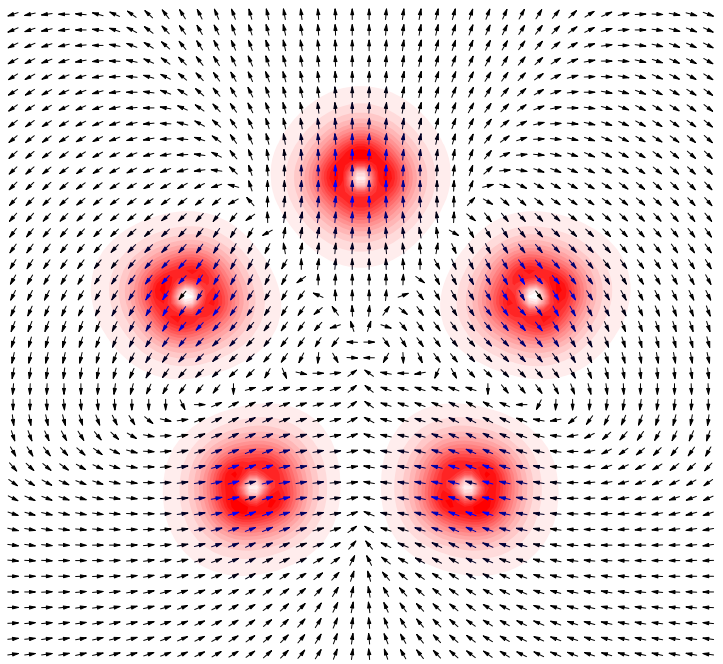

(b)

FIG. 3: Magnetization plot for $\mathrm{Cr}_{5}$ obtained in a PBE calculation. The arrows show the direction of the spin polarization $(\mathbf{m} / m)$ in the plane containing the nuclei, whereas the spin modulus $m$ is represented in red. The top (a) and bottom (b) panels show two energetically degenerate configurations with + and - chiralities, respectively.

we would like to recall that the Cr cluster geometries are fixed in these test calculations and hence relaxation effects are not included in the reported atomic magnetic moments. For $\mathrm{Cr}_{3}$ and $\mathrm{Cr}_{5}$, we obtain comparable values of the atomic magnetization $m$ for a given functional. This is not the case for $\mathrm{Cr}_{7}$ and $\mathrm{Cr}_{12}$ clusters, where the atomic magnetic moments of the internal $\mathrm{Cr}$ atoms are smaller than those of the external atoms in all cases. From the values of $m_{c}$ and $m_{e}$ (see Fig. 1) for $\mathrm{Cr}_{7}$ with PBEh and GUHF, we can notice a large effect of Hartree-

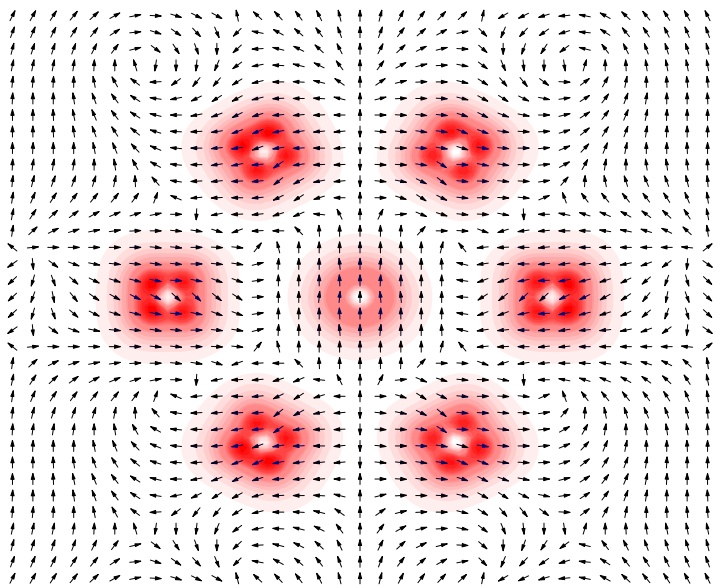

FIG. 4: Magnetization plot for the $\mathrm{Cr}_{7}$ cluster obtained in a PBE calculation. The arrows show the direction of the spin polarization $(\mathbf{m} / m)$ in the plane containing the nuclei, whereas the spin modulus $m$ is represented in red.

Fock exchange compared to the rest of the clusters. In fact, GUHF gives an atomic magnetization in $\mathrm{Cr}_{7}$ approximately 10 times larger than those obtained with LSDA, while for $\mathrm{Cr}_{3}$ and $\mathrm{Cr}_{5}$ the ratio is less than 2, and for $\mathrm{Cr}_{12}$ is about 3 .

In Figs. 4 and 5, we present the PBE spin density in the plane containing the nuclei for $\mathrm{Cr}_{7}$ and $\mathrm{Cr}_{12}$, respectively. The plot for $\mathrm{Cr}_{7}$ resembles the spin density in the usupported $\mathrm{Cr}$ monolayer shown in Ref. 12. The difference arises mainly in the low spin polarization region of the $\mathrm{Cr}_{7}$ cluster. $\mathrm{Cr}_{12}$ can be thought as a cluster model of a two-dimensional Kagomé lattice. However, as the magnetization of the internal and external atoms is not the same, one would expect that the actual ground state is a result of different competing effects. For instance, in a Heisenberg spin Hamiltonian, the uppermost Cr atom, Fig. 1 1 , couples antiferromagnetically with its weaker polarized nearest neighbors and with its stronger polarized second nearest neighbors, and it is not clear $a$ priori which coupling is larger. Therefore, we expect that this type of noncollinear DFT calculations would be helpful to investigate the magnetic properties of clusters and molecules where a simple Heisenberg spin Hamiltonian cannot be straightforwardly applied.

\section{SUMMARY AND CONCLUSIONS}

We have generalized the treatment of the electronic spin degrees of freedom in density functional calculations to the case where the vector variables employed in the definition of the $\mathrm{XC}$ energy can vary in any direction. Our noncollinear generalization can be applied to general functionals containing a variety of ingredients. Our 


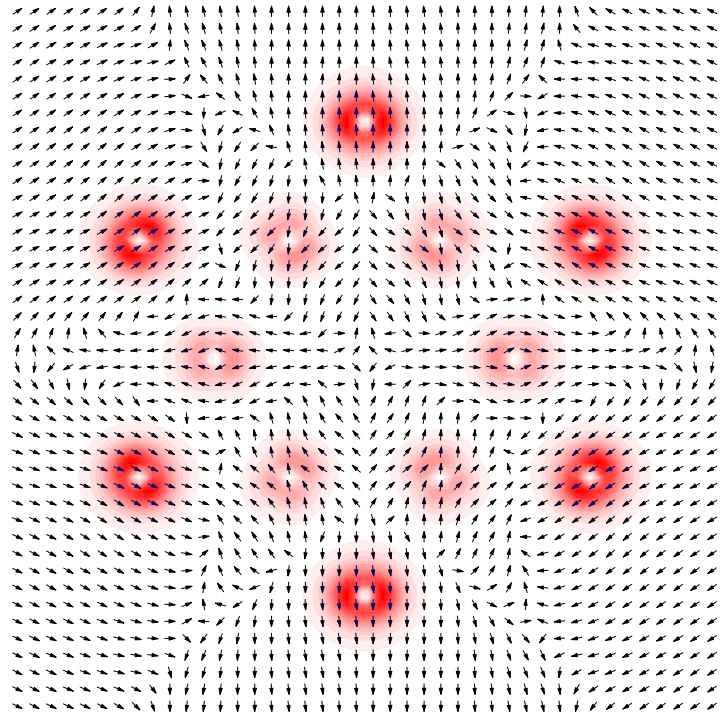

FIG. 5: Magnetization plot for the $\mathrm{Cr}_{12}$ cluster obtained ina PBE calculation. The arrows show the direction of the spin polarization $(\mathbf{m} / m)$ in the plane containing the nuclei, whereas the spin modulus $m$ is represented in red. generalization assumes that the $\mathrm{XC}$ energy depends on the local variables in the same manner as in the standard collinear case, and that the energy expression is invariant under rigid rotations of the spin quantization axis. This is not the most general way to define energy functionals for noncollinear magnetic systems, but it provides a general starting point to incorporate new terms like those suggested in Refs. 19 and 21.

Test calculations on planar $\mathrm{Cr}$ clusters suggest that the choice of energy functional has an important impact on the resulting atomic magnetic moments, giving qualitatively similar but quantitatively different results. We expect that our generalization will open the door to studies on the performance of density functionals other than LSDA for noncollinear magnetic systems.

\section{ACKNOWLEDGEMENTS}

J.E.P thanks K. Capelle, R. Pino, A. Izmailov, and O. Hod for useful discussions and T. Van Voorhis for suggesting the $\mathrm{Cr}_{12}$ example. This work was supported by the Department of Energy Grant No. DE-FG0201ER15232, ARO-MURI DAAD-19-3-1-0169, and the Welch Foundation.
1 U. von Barth and L. Hedin, J. Phys. C 5, 1629 (1972).

2 P. Hohenberg and W. Kohn, Phys. Rev. B 136, 864 (1964).

3 W. Kohn and L. J. Sham, Phys. Rev. A 140, 1133 (1965).

${ }^{4}$ Y. Tsunoda, J. Phys.: Condens. Matter 1, 10427 (1989).

${ }^{5}$ E. Sjöstedt and L. Nordström, Phys. Rev. B 66, 014447 (2002).

6 D. Grohol, K. Matan, J.-H. Cho, S.-H. Lee, J. W. Lynn, D. G. Nocera, and Y. S. Lee, Nature Materials 4, 323 (2005).

7 G. Venturini, R. Welter, E. Ressouche, and B. Malaman, J. Alloys Compd. 210, 213 (1994).

8 M. Uchida, Y. Onose, Y. Matsui, and Y. Tokura, Science 311, 359 (2006).

9 J. Kübler, K.-H. Höck, J. Sticht, and A. R. Williams, J. Phys. F: Met. Phys. 18, 469 (1988).

10 L. Nordsröm and D. J. Singh, Phys. Rev. Lett. 76, 4420 (1996).

11 T. Oda, A. Pasquarello, and R. Car, Phys. Rev. Lett. 80, 3622 (1998).

12 P. Kurtz, F. Förster, L. Nordsröm, G. Bihlmayer, and S. Blügel, Phys. Rev. B 69, 024415 (2004).

13 S. Yamanaka, D. Yamaki, Y. Shigeta, H. Nagao, Y. Yoshioka, N. Suzuki, and K. Yamaguchi, Int. J. Quantum Chem. 80, 664 (2000).

14 C. Kohl and G. F. Bertsch, Phys. Rev. B 60, 4205 (1999).

15 R. C. Longo, E. G. Noya, and L. J. Gallego, Phys. Rev. B 72, 174409 (2005).

16 J. Mejía-López, A. H. Romero, M. E. Garcia, and J. L. Morán-López, Phys. Rev. B 74, 140405(R) (2006).

17 J. P. P. A. Ruzsinszky, J. Tao, V. N. Staroverov, G. E. Scuseria, and G. I. Csonka, J. Chem. Phys. 123, 062201
(2005).

18 A. D. Becke, J. Chem. Phys. 98, 5648 (1993).

19 L. Kleinman, Phys. Rev. B 59, 3314 (1999).

20 J. A. Pople, P. M. W. Gill, and B. G. Johnson, Chem. Phys. Lett. 199, 557 (1992).

21 K. Capelle, G. Vignale, and B. L. Györffy, Phys. Rev. Lett. 87, 206403 (2001).

${ }^{22}$ K. Knopfle, L. M. Sandratskii, and J. Kubler, Phys. Rev. B 62, 5564 (2000).

23 R. McWeeny, Methods of Molecular Quantum Mechanics (Academic Press, San Diego, CA, 1989).

${ }^{24}$ Gaussian Development Version, Revision E.05, M. J. Frisch, G. W. Trucks, H. B. Schlegel, G. E. Scuseria, M. A. Robb, J. R. Cheeseman, J. A. Montgomery, Jr., T. Vreven, G. Scalmani, K. N. Kudin, S. S. Iyengar, J. Tomasi, V. Barone, B. Mennucci, M. Cossi, N. Rega, G. A. Petersson, H. Nakatsuji, M. Hada, M. Ehara, K. Toyota, R. Fukuda, J. Hasegawa, M. Ishida, T. Nakajima, Y. Honda, O. Kitao, H. Nakai, X. Li, H. P. Hratchian, J. E. Peralta, A. F. Izmaylov, E. Brothers, V. Staroverov, R. Kobayashi, J. Normand, J. C. Burant, J. M. Millam, M. Klene, J. E. Knox, J. B. Cross, V. Bakken, C. Adamo, J. Jaramillo, R. Gomperts, R. E. Stratmann, O. Yazyev, A. J. Austin, R. Cammi, C. Pomelli, J. W. Ochterski, P. Y. Ayala, K. Morokuma, G. A. Voth, P. Salvador, J. J. Dannenberg, V. G. Zakrzewski, S. Dapprich, A. D. Daniels, M. C. Strain, O. Farkas, D. K. Malick, A. D. Rabuck, K. Raghavachari, J. B. Foresman, J. V. Ortiz, Q. Cui, A. G. Baboul, S. Clifford, J. Cioslowski, B. B. Stefanov, G. Liu, A. Liashenko, P. Piskorz, I. Komaromi, R. L. Martin, D. J. Fox, T. Keith, M. A. Al-Laham, C. Y. Peng, A. Nanayakkara, M. Challa- 
combe, W. Chen, M. W. Wong, and J. A. Pople, Gaussian, Inc., Wallingford CT, 2006. Gaussian, Inc., Pittsburgh PA, 2003.

25 P. Pulay, Chem. Phys. Lett. 73, 393 (1980).

${ }^{26}$ K. N. Kudin, G. E. Scuseria, and E. Cancès, J. Chem. Phys. 116, 8255 (2002).

27 M. Dolg, U. Wedig, H. Stoll, and H. Preuss, J. Chem. Phys. 86, 866 (1987).

28 S. H. Vosko, L. Wilk, and M. Nusair, Can. J. Phys 58, 1200 (1980).

29 J. P. Perdew, K. Burke, and M. Ernzerhof, Phys. Rev.
Lett. 77, 3865 (1996).

30 J. Tao, J. P. Perdew, V. N. Staroverov, and G. E. Scuseria, Phys. Rev. Lett. 91, 146401 (2003).

31 J. P. Perdew, M. Ernzerhof, and K. Burke, J. Chem. Phys. 105, 9982 (1997).

32 M. Ernzerhof and G. E. Scuseria, J. Chem. Phys. 110, 5029 (1999).

33 C. Adamo and V. Barone, J. Chem. Phys. 110, 6158 (1999). 